\title{
A note about the delta-moment in ARMA-APARCH models with stable conditional distributions and GEV.
}

\section{Una nota sobre el delta- simo momento en modelos ARMA-APARCH con distribuciones condicionales estables y GEV.}

\author{
Thiago R. Sousa *, Cira E. G. Otiniano ${ }^{\dagger}$, and Silvia R. C. Lopes ${ }^{\ddagger}$ \\ Received, Feb. 13, 2018 \\ Accepted, Jun. 06, 2018
}

DOI: http://dx.doi.org/10.17268/sel.mat.2018.01.02

\begin{abstract}
In a ARMA-APARCH time series model with innovations $Z$, the delta-stationarity condition of the APARCH process involves the delta-th moment of the difference between the absolute value of the innovations with the product of the asymmetry parameter and the innovations. This moment allows calculating more efficiently the estimates of the parameters of the model by maximum likelihood. In this article, we obtain explicit expressions of this delta - th moment where Z has stable and GEV distribution. These moments have been implemented in our GEVStableGarch package available in CRAN R-PROJECT developed to estimate the parameters of ARMA-GARCH / APARCH models with stable innovations and GEV.
\end{abstract}

Keywords. ARMA, GARCH, APARCH, Stationarity, Stable distribution, GEV distribution.

\section{Resumen}

En un modelo de sries temporales ARMA-APARCH con innovaciones Z, la condicin de delta - estacionariedad del proceso APARCH envuelve el delta - simo momento de la diferencia entre el valor absoluto de las innovaciones con el producto del parmetro de asimetra y las innovaciones. Este momento permite calcular de forma mas eficiente las estimativas de mxima verosimilitud de los parmetros del modelo. En este artculo, son obtenidas expresiones explcitas de ese delta - simo momento onde $Z$ tem distribucin estable y GEV. Essos momentos se han implementado en nuestro paquete GEVStableGarch disponible en CRAN R-PROJECT desarrollado para estimar los parmetros de los modelos ARMA-GARCH / APARCH con innovaciones estables y GEV.

Palabras clave. ARMA, GARCH, APARCH, Estacionalidad, Distribucin estable, Distribucin GEV.

1. Introduction. Modeling financial time series is an attempt to find satisfactory statistical models to explain what is observed empirically. According to Jondeau et al. (2007) some of the stylized facts about financial data are: the unconditional distribution of returns is heavy tailed and asymmetric, and they exhibit volatility clustering, i.e., a larger return is usually followed by another large return. This serial correlation presented in financial data was successfully modeled by Engle (1982) within his ARCH (autoregressive conditional heteroskedasticity) model later generalized by Bollerslev (1986) in their GARCH (Generalized Autoregressive Conditional Heteroskedastic) model. The ARCH class of models has seen widespread application of its various extensions found in the literature. A last extension is the APARCH (asymmetric power ARCH), introduced by Ding et al. (1993).

Modeling financial data with a simple ARMA process does not give good results, because in general the sample auto-correlation of the squared residuals is significantly different from zero. The approach that has proven to be successful to account for both time dependence and serial correlation consists in combining

\footnotetext{
*Departamento de Estatstica, UnB, 70910-900 Braslia, DF, Brazil (thiagoestatistico@gmail. com)

${ }^{\dagger}$ Departamento de Estatstica, UnB, 70910-900 Braslia, DF, Brazil (cira@unb . br )

${ }_{\ddagger}^{\ddagger}$ Departmento de Matemtica,Universidade Federal de Rio Grande do Sul, Brazil (silviarc. lopes@gmail.com ). This work is licensed under the Creative Commons Attribution-NoComercial-ShareAlike 4.0.
} 
the ARMA and GARCH/APARCH equations in the same model (see, for instance, Nakatsuma (2000); Brummelhuis and Kaufmann (2007); Wuertz et al. (2009) and Zhao et al. (2011)).

A process $\left\{X_{t}\right\}_{t \in \mathbb{Z}}$ is an $\operatorname{ARMA}(m, n)-\operatorname{APARCH}(p, q)$ model if $X_{t}$, for all $t \in \mathbb{Z}$, satisfies:

$$
\begin{aligned}
X_{t} & =\sum_{i=1}^{m} a_{i} X_{t-i}+\sum_{j=1}^{n} b_{j} \varepsilon_{t-j}+\varepsilon_{t}, \\
\varepsilon_{t} & =Z_{t} \sigma_{t} \quad Z_{t} \stackrel{i . i . d .}{\sim} \mathcal{D}_{\vartheta}(0,1), \\
\sigma_{t}^{\delta} & =\omega+\sum_{i=1}^{p} \alpha_{i}\left(\left|\varepsilon_{t-i}\right|-\gamma_{i} \varepsilon_{t-i}\right)^{\delta}+\sum_{j=1}^{q} \beta_{j} \sigma_{t-j}^{\delta},
\end{aligned}
$$

where $a_{1}, \ldots, a_{m}$ are the autoregressive coefficients, $b_{1}, \ldots, b_{n}$ are the moving average coefficients and the APARCH (The asymmetric power ARCH) parameters are $\omega>0, \alpha_{1}, \ldots, \alpha_{p} \geq 0, \beta_{1}, \ldots, \beta_{q} \geq 0,-1<$ $\gamma_{1}, \ldots, \gamma_{p}<1$ and $\delta>0$. $\mathcal{D}_{\vartheta}(0,1)$ is distribution of the innovations with zero location and unit scale and $\vartheta$ are the parameters that describe the skew and the shape of the distribution.

ARMA and GARCH models are particular cases of (1.1). If all the coefficients $\alpha$ and $\beta$ are zero we get a pure $\operatorname{ARMA}(m, n)$ model and if all the coefficients $a$ and $b$ are zero, $\gamma$ zero and $\delta=2$ we get a pure $\operatorname{GARCH}(\mathrm{p}, \mathrm{q})$ model.

In practice, the application of ARMA-GARCH models is proved to generate best results when the conditional distribution has some connection with the nature of the data. For example, Zhao et al. (2011) used an AR(1)-GARCH(1,1) model with conditional GEV distribution for modeling maximum daily returns. The results showed that this combination can capture and explain extreme quantiles better than the standard model with normal distribution. Stable distributions are also useful for modeling heavy tailed data, which is usually the case of stock market and foreign exchange rate data (see Nolan (2001)). Thus, combining ARMA- GARCH/APARCH models with stable distributions gives us a powerful tool for modeling financial data (see Mittnik and Paolella (2000), Curto et al. (2008) and Frain (2009)).

The estimation of general ARMA-GARCH/APARCH models with conditional GEV or stable distributions are available in our GEVStableGarch-2015 package. It contains functions for estimating and simulating ARMA-GARCH or APARCH models with conditional stable and GEV distributions. The stationarity conditions of the models are usually ignored in practice, but in many real life problems the researcher is interested in finding a stationary solution. In our algorithms we implemented the $\delta$ - stationarity conditions for a more efficient and fast estimation.

The $\delta$ - order stationarity conditions of a APARCH model was showed by Ling (2002). This result states that there exists a unique $\delta$-order stationary solution of the APARCH model, if and only if $\sum_{i=1}^{p} \lambda_{i} \alpha_{i}+$ $\sum_{j=1}^{q} \beta_{j}<1$, where $\lambda_{i}=E\left(\left|Z_{t}\right|-\gamma_{i} Z_{t}\right)^{\delta} \quad(\delta$-moment $)$.

In this paper we review the stationarity conditions for ARMA-GARCH/APARCH models with conditional GEV or stable distributions and explicit expressions of $\lambda_{i}$, where $Z$ has stable distribution and GEV, are obtained. Also, we present two case study.

The remainder of the paper is structured as follows. In Section 2, we review stable and GEV distributions. In Section 3, $\delta$ - moments in which $Z_{t}$ has stable and GEV distributions are derived. In Section 4, two case study were added.

2. Stationarity conditions of the ARMA-APARCH model. In this section, first we review the stable and GEV distribution.

2.1. Stable and GEV distributions. Stable distributions are used to analyze asset returns such as exchange rates and stock prices. Theory and applications of stable distributions can be found in the book of Samorodnitsky and Taqqu (1994).

Non-degenerate $Z$ is stable if and only if for all $n>1$, there exist constant $d_{n} \in R$ such that

$$
Z_{1}+\cdots+Z_{n}=n^{1 / \alpha} Z+d_{n}
$$

where $Z_{1}, \ldots, Z_{n}$ are independent, identical copies of $Z$ and $\alpha \in(0,2]$. $Z$ is strictly stable if and only if $d_{n}=0$ for all $n$.

A stable random variable is usually known by their characteristic functions, because there is no simple closed form for the probability density function (pdf). In the next section we present an expression for this density in terms of the function $\mathrm{H}$.

The characteristic function of a random variable stable $Z$ with $\mu \in \mathbb{R}$ location parameter, $\sigma>0$ scale parameter, $-1 \leq \beta \leq 1$ skewnes parameter and $0<\alpha \leq 2$ shape parameter know as index of stability, is given by 


$$
\phi_{X}(t)=\left\{\begin{array}{l}
\exp \left\{i \mu t-\sigma^{\alpha}|t|^{\alpha}\left[1-i \beta \frac{t}{|t|} \tan \left(\frac{\pi \alpha}{2}\right)\right]\right\}, \alpha \neq 1 \\
\exp \left\{i \mu t-\sigma^{\alpha}|t|\left[1+i \beta \frac{t}{|t|} \frac{2}{\pi} \ln |t|\right]\right\}, \alpha=1 .
\end{array}\right.
$$

When $\beta=0$, the stable random variable is symmetric. $Z$ is Gaussian for $\alpha=2$, Cauchy for $\alpha=1$ and Lvy for $\alpha=1 / 2$. We will denote stable Lvy distribution by $S(\alpha, \beta, \sigma, \mu)$ when $0<\alpha<2$. In this case, the moment $E\left(Z^{r}\right)$ is infinite for $r<\alpha$; that is $Z$ has infinite second moment and has finite mean for $\alpha>1$.

In Fig. 2.1 we show densities of a variable stable. In each sub figure we vary a parameter.
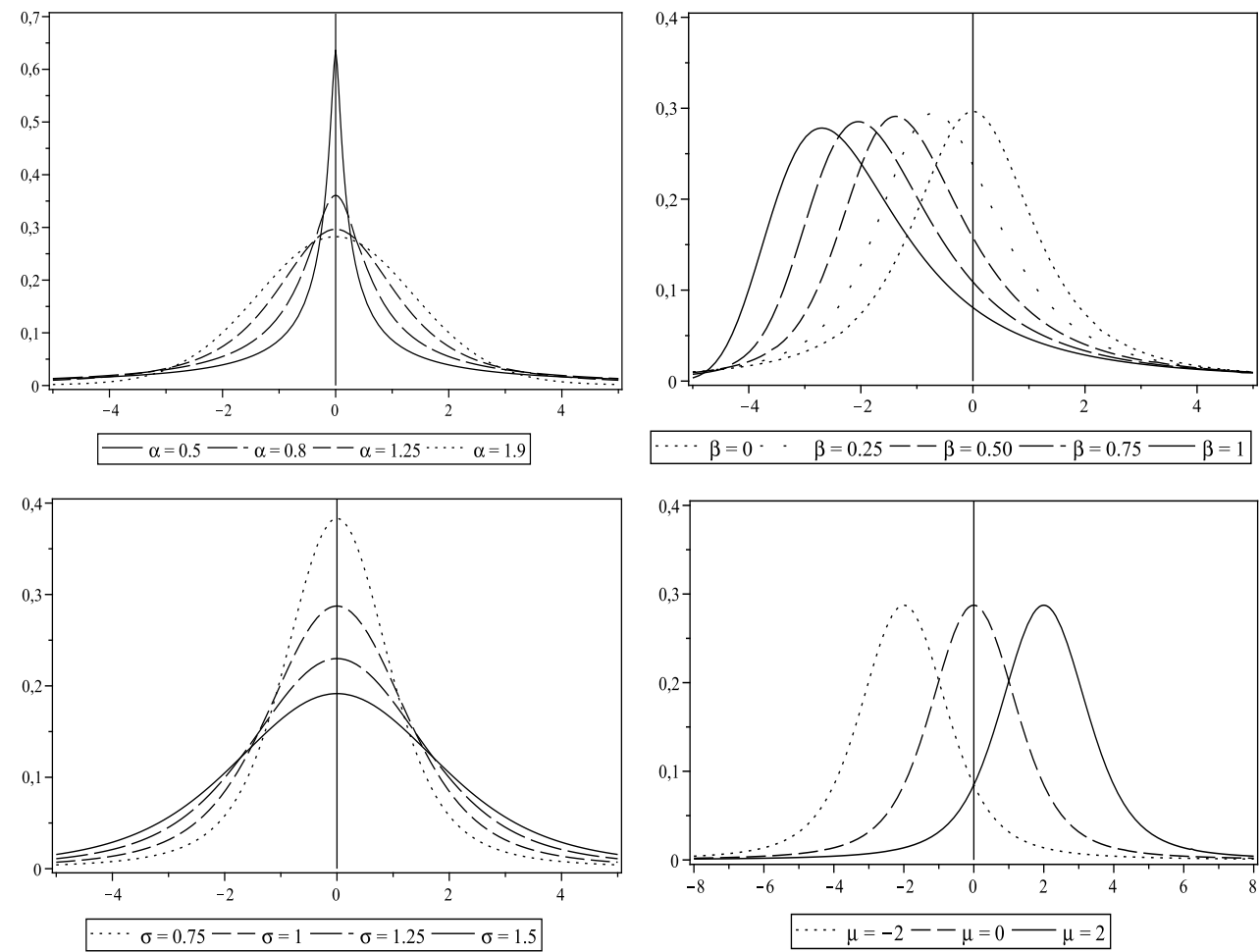

Figure 2.1. Density correspond to $S(\alpha, 0,1,0), S(3 / 2, \beta, 1,0), S(3 / 2,0, \sigma, 0)$ and $S(3 / 2,0,1, \mu)$ in each sub figure, respectively.

On the other hand, three distributions arise naturally when working with the distribution of maximum or minimum data, they are called extreme value distributions . An extreme value distribution (EVD), defined by Fisher and Tippett (1928) and Gnedenko (1943), is the non degenerate limit distribution of the standardized maximum of independent and identically distributed (i.i.d.) random variables.

To define the EVD, let $Z_{1}, \ldots, Z_{n}$ be an i.i.d. sequence of random variables. If there are normalizing constants $a_{n}>0, b_{n} \in R$ such that

$$
\lim _{n \rightarrow \infty} P\left(\frac{\max \left\{Z_{1}, \ldots, Z_{n}\right\}-b_{n}}{a_{n}} \leq x\right)=F(x),
$$

with $F(x)$ nondegenerate distribution, then $F$ is an EVD. In this case, $F$ belongs to one of the following three types of cumulative distribution functions:

Frchet: $\Phi(x ; \alpha, \sigma, \mu)= \begin{cases}\exp \left[-\left(\frac{x-\mu}{\sigma}\right)^{-\alpha}\right], & x \geq \mu \\ 0, & x<\mu\end{cases}$ 


$$
\begin{aligned}
& \text { Weibull: } \Psi(x ; \alpha, \sigma, \mu)= \begin{cases}\exp \left[-\left(-\frac{x-\mu}{\sigma}\right)^{\alpha}\right] & , x<\mu \\
0 & , x>\mu ;\end{cases} \\
& \text { Gumbel: } \Lambda(x ; \sigma, \mu)=\exp \left[-\exp \left(-\frac{x-\mu}{\sigma}\right)\right], \forall x \in \mathbb{R},
\end{aligned}
$$

where $\alpha>0$ shape parameter, $\sigma>0$ scale parameter, and $\mu \in \mathbb{R}$ location parameter.

By Jenkinson (1955), a generalized extreme value (GEV) distribution, denoted by $\mathrm{GEV}(\xi, \sigma, \mu)$, includes these three distributions and its density function is given by:

$$
f_{Z}(z ; \xi, \sigma, \mu)= \begin{cases}{\left[1+\xi\left(\frac{z-\mu}{\sigma}\right)\right]^{-1-1 / \xi} \exp \left[-\left(1+\xi\left(\frac{z-\mu}{\sigma}\right)\right)^{-1 / \xi}\right],} & \text { if } \xi \neq 0 \\ \exp \left(-\left(\frac{z-\mu}{\sigma}\right)\right) \exp \left[-\exp \left(-\left(\frac{z-\mu}{\sigma}\right)\right)\right], & \text { if } \xi=0\end{cases}
$$

where $\mu \in \mathbb{R}$ is the location parameter, $\sigma>0$ is the scale parameter and $\xi \in \mathbb{R}$ is the shape parameter (known as tail index). The support of $f_{Z}$ is the interval in $\mathbb{R}$ such that $1+\xi(z-\mu) / \sigma>0$.

The mean of the GEV distribution exists whenever the shape parameter $\xi<1$ and its second moment is infinite when $\xi \geq 1 / 2$.

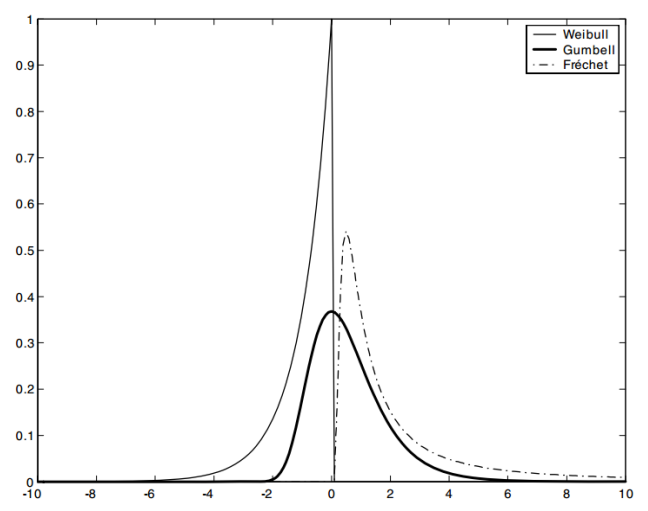

FIGURE 2.2. EVD densities.

2.2. Stationarity. Since we want to estimate the parameters that better adjust real data to ARMAAPARCH models, the notion of stationarity is crucial. Usually, even when the data set is non-stationary in appearance, we still are able to apply transformation techniques so that the resulting time series can be reasonably modeled as a stationary process (see Brockwell (1991)).

The stationarity property of the combined ARMA-APARCH model is achieved when both ARMA and APARCH models are individually stationary. Usually, if the conditional distribution we are dealing with has infinite variance, it is only used in the domain where its variance remains finite. For example, the standard Student's t distribution with $\nu$ degrees of freedom is defined for any $\nu>0$, but the ARMA-APARCH model with conditional Student's t distribution described in Wurtz (2006) is defined only for $\nu>2$ in which case the variance of the Student's t distribution is finite.

The stationarity of the ARMA model is intimately connected with the roots of the polynomials $a(z)=$ $1-a_{1} z-\cdots-a_{m} z^{m}$ and $b(z)=1-b_{1} z-\cdots-b_{n} z^{n}$ representing the ARMA part of Definition 1.1. In many cases the conditional distribution of the model has a finite variance and therefore, the condition for the existence of a stationary solution is just that $a(z)=1-a_{1} z-\cdots-a_{m} z^{m}$ has no roots for all $z \in \mathbb{C}$ such that $|z|=1$. On the other hand, if the distribution has an infinite variance the conditions for the existence of stationary solutions becomes (see Brockwell (1985)),

$$
a(z) b(z) \neq 0, \text { for all } z \in \mathbb{C}, \text { such that }|z| \leq 1
$$


The stationarity of APARCH models has a historical background that is worth exploring. In the context of a model where the innovations have a finite variance, Bollerslev (1986) proved the second order stationarity of the GARCH(p,q) model. Then, Nelson (1990) demonstrated the strict stationarity of the empirically important case GARCH(1,1). His results were then generalized by Bougerol (1992) for the GARCH(p,q) model. Finally, Ling (2002) showed which conditions the APARCH(p,q) model would be $\delta$-order stationary. This last result states that there exists a unique $\delta$-order stationary solution of the APARCH model if and only if

$$
\sum_{i=1}^{p} \lambda_{i} \alpha_{i}+\sum_{j=1}^{q} \beta_{j}<1
$$

where

$$
\lambda_{i}=E\left(\left|Z_{t}\right|-\gamma_{i} Z_{t}\right)^{\delta}, Z_{t} \sim \mathcal{D}_{\vartheta}(0,1)
$$

The case of infinite variance has a somewhat different historical background. It is worth noting that the theoretical results were all based on the important work of Bougerol (1992). The first assumption made on stable distributions is that the index of stability $\alpha$ must be greater than one, because in this case the innovations have finite first moment. According to Mittnik (1995), this assumption seems plausible since most financial time series have finite mean. The second assumption is that they must have a $\delta$-moment finite, which means that we must restrict our model to $1<\delta<\alpha$.

Regarding the stationarity of these models, Mittnik (1995) proved the strict stationarity of the stable $\operatorname{GARCH}(\mathrm{p}, \mathrm{q})$ model, $Z_{t} \sim S(\alpha, \beta, 1,0)=S(\alpha, \beta ; 1)$. Then, Mittnik (2002) derived conditions for the strict stationarity of the $\operatorname{APARCH}(\mathrm{p}, \mathrm{q})$ model with all coefficients $\gamma_{i}=0$ (namely the power-GARCH model). Finally, Diongue (2008) showed that the general APARCH(p,q) model has a strictly stationary solution if and only if (2.4) and (2.5) are satisfied, however, he did not get an explicit expression for $\lambda_{i}$.

The same stationarity conditions, (2.4) and (2.5), are valid for an APARCH(p,q) model with $Z_{t} \sim$ $\operatorname{GEV}(\xi, 1,0)$.

The stationarity conditions of the ARMA part of the model are easy to implement computationally, but if we want to impose stationarity of the APARCH equation the problem of efficiency arises. The main problem is that equations (2.4) and (2.5) depend on $\lambda_{i}$. However, their computation is faster when they have an explicit expression.

Figure 1.1. shows the result of the simulation of 1000 observations of the AR(1)-GARCH(1,1) model with parameters vector $\phi=\left(b_{1}, a_{1}, \alpha_{1}, \beta_{1}, \psi\right)=(0.2,0.3,0.2,0.3,0.2,0.2)$ and GEV and normal innovations distributions.

Figure 1.2. shows the result of the simulation of 1000 observations of the AR(1)-GARCH(1,1) model with stable and normal innovations when $\phi=\left(b_{1}, a_{1}, \alpha_{1}, \beta_{1}, \alpha, \beta,\right)=(0.2,0.3,0.2,0.3,0.2,1.8,0)$ and stable and normal innovations distributions.
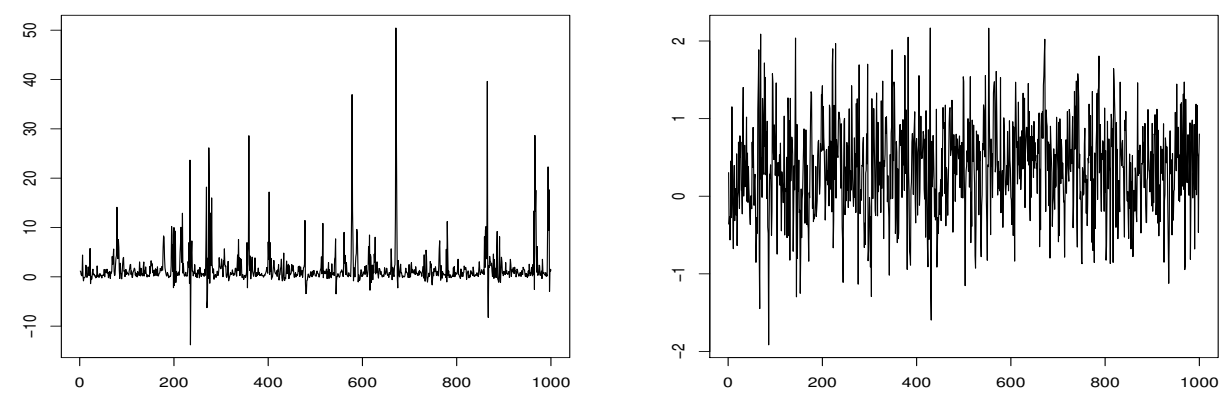

Figure 2.3. Left side: AR (1) -GARCH (1.1) with $Z_{t} \sim G E V$. Right side: AR (1) -GARCH (1,1) with $Z_{t} \sim$ Normal

3. $\delta$ - moments. In this section, the $\delta$-moment defined in (2.5) of the APARCH(p,q) model with $Z \sim$ $S(\alpha, \beta, 1)$ and $Z \sim G E V(\xi, 1,0)$ are derived.

Firstly, in the Proposition 1, we obtain the $\delta$-moment for $Z \sim S(\alpha, \beta, 1)$. For this, we have that when $\alpha \neq 1$, the characteristic function (2.1) can be too written as

$$
\phi_{X}(t)=\exp \left\{-\tilde{\sigma}^{\alpha}|t|^{\alpha} e^{-i \tilde{\beta}(\operatorname{sign} t) \frac{\pi}{2} K(\alpha)}+i \mu t\right\},
$$



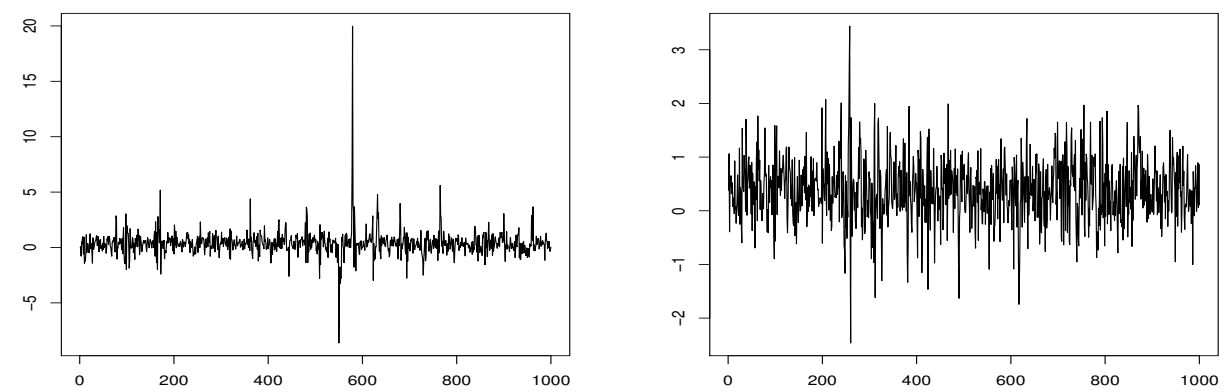

FIGURE 2.4. Left side: $A R(1)-G A R C H(1.1)$ with $Z_{t} \sim S_{\alpha}$. Right side: AR(1)-GARCH (1,1) with $Z_{t} \sim$ Normal

where

$$
\tilde{\sigma}=\sigma\left(1+\beta^{2} \tan ^{2} \alpha \frac{\pi}{2}\right)^{1 / 2 \alpha}
$$

$$
K(\alpha)=\left\{\begin{array}{lll}
\alpha, & \text { if } \quad \alpha<1 \\
\alpha-2, & \text { if } \quad \alpha>1
\end{array}\right.
$$

and

$$
\tilde{\beta}= \begin{cases}\frac{2}{\pi \alpha} \operatorname{Arctan}\left(\beta \tan \alpha \frac{\pi}{2}\right), & \text { if } \quad 0<\alpha<1 \\ \frac{2}{\pi(\alpha-2)} \operatorname{Arctan}\left(\beta \tan \frac{\pi(\alpha-2)}{2}\right), & \text { if } 1<\alpha<2 .\end{cases}
$$

Schneider (1986) gave a representation of stable Lvy density, in terms of the $\mathrm{H}$ - function. Expressions equivalent to the ones obtained by Schneider (1986) for the pdf of $S(\alpha, \beta, \sigma, \mu)$, with $0<\alpha<2, \alpha \neq 1$, are:

$$
\left.f(x ; \alpha, \tilde{\beta}, \mu, \tilde{\sigma})=\frac{1}{\alpha \tilde{\sigma}} H_{2}^{1} \underset{2}{\frac{x-\mu}{\tilde{\sigma}}} \mid \begin{array}{c}
\left(1-\frac{1}{\alpha}, \frac{1}{\alpha}\right),\left(\frac{1}{2}-\frac{\tilde{\beta} K(\alpha)}{2 \alpha}, \frac{1}{2}+\frac{\tilde{\beta} K(\alpha)}{2 \alpha}\right) \\
(0,1),\left(\frac{1}{2}-\frac{\tilde{\beta} K(\alpha)}{2 \alpha}, \frac{1}{2}+\frac{\tilde{\beta} K(\alpha)}{2 \alpha}\right)
\end{array}\right]
$$

for $x \geq \mu$, and

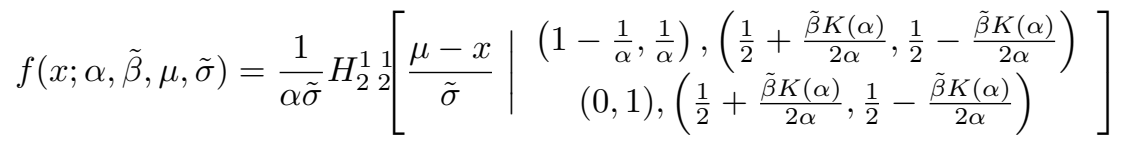

for $x<\mu$. The function $H_{2,2}^{1,1}(z)$ is the H-function defined in Mathai et al. (2010), as

$$
\begin{aligned}
& H_{p, q}^{m, n}(z)=H_{p, q}^{m, n}\left[z \mid \begin{array}{cccccc}
\left(a_{1}, A\right), & \ldots, & \left(a_{n}, A_{n}\right), & \left(a_{n+1}, A_{n+1}\right), & \ldots, & \left(a_{p}, A_{p}\right) \\
\left(b_{1}, B_{1}\right), & \ldots, & \left(b_{m}, B_{m}\right), & \left(b_{m+1}, B_{m+1}\right), & \ldots, & \left(b_{q}, B_{q}\right)
\end{array}\right] \\
& =\frac{1}{2 \pi i} \int_{L} \Xi_{p, q}^{m, n}(s) z^{-s} d s
\end{aligned}
$$

where

$$
\Xi_{p, q}^{m, n}(s)=\frac{\prod_{j=1}^{m} \Gamma\left(b_{j}+B_{j} s\right) \prod_{j=1}^{n} \Gamma\left(1-a_{j}-A_{j} s\right)}{\prod_{j=m+1}^{q} \Gamma\left(1-b_{j}-B_{j} s\right) \prod_{j=n+1}^{p} \Gamma\left(a_{j}+A_{j} s\right)}
$$

and $A_{j}$ and $B_{j}$ are assumed to be positive quantities and all the $a_{j}$ and $b_{j}$ are complex. The contour $L$ runs from $c-i \infty$ to $c+i \infty$ such that the poles of $\Gamma\left(b_{j}+B_{j} s\right), j=1, \ldots, m$ lie to the left of $L$ and the poles of $\Gamma\left(1-a_{j}-A_{j} s\right), j=1, \ldots, n$ lie to the right of $L$. 
To compute the $\delta$-moment of the $\operatorname{APARCH}(\mathrm{p}, \mathrm{q})$ model with $Z \sim S(\alpha, \beta, 1)$ we used the Mellin transform of the stable density.

In general, the Mellin transform of a $f(x)$ real function is uniquely defined almost everywhere for $x>0$ and that is absolutely integrable over the interval $(0,+\infty)$, thus its Mellin transform exists and is defined as (see Mathai et al. (2010) ):

$$
M_{s} f(x)=\int_{0}^{+\infty} x^{s-1} f(x) d x
$$

Proposition 1. If $Z \sim S(\alpha, \beta ; 1)$ then the $\delta$-moment $E(|Z|-\gamma Z)^{\delta}$ is given by

$$
\begin{aligned}
E(|Z|-\gamma Z)^{\delta} & =\frac{(1-\gamma)^{\delta} \tilde{\sigma}^{\delta} \Gamma(\delta+1) \Gamma\left(-\frac{\delta}{\alpha}\right)}{\alpha \Gamma\left[\left(\frac{1}{2}+\tilde{\beta} \frac{k(\alpha)}{2 \alpha}\right)(-\delta)\right] \Gamma\left[\frac{1}{2}-\tilde{\beta} \frac{k(\alpha)}{2 \alpha}+\left(\frac{1}{2}+\tilde{\beta} \frac{k(\alpha)}{2 \alpha}\right)(\delta+1)\right]} \\
+ & \frac{(1+\gamma)^{\delta} \Gamma(\delta+1) \Gamma\left(-\frac{\delta}{\alpha}\right)}{\alpha \tilde{\sigma} \Gamma\left[\left(\frac{1}{2}-\tilde{\beta} \frac{k(\alpha)}{2 \alpha}\right)(-\delta)\right] \Gamma\left[\frac{1}{2}+\tilde{\beta} \frac{k(\alpha)}{2 \alpha}+\left(\frac{1}{2}-\tilde{\beta} \frac{k(\alpha)}{2 \alpha}\right)(\delta+1)\right]},
\end{aligned}
$$

where $\tilde{\sigma}, k(\alpha)$ and $\tilde{\beta}$ are given in (3.2), (3.3) and (3.4), respectively. Proof: By definition

$$
E(|Z|-\gamma Z)^{\delta}=\int_{-\infty}^{\infty}(|z|-\gamma z)^{\delta} f_{Z}(z ; \alpha, \tilde{\beta}, \tilde{\sigma}, 0) \mathrm{d} z
$$

To applied the Mellin transform (3.9) we rewrite (3.11) as

$$
\begin{aligned}
E(|Z|-\gamma Z)^{\delta} & =(1+\gamma)^{\delta} \int_{0}^{\infty} z^{\delta} f_{Z}(-z ; \alpha, \tilde{\beta}, \tilde{\sigma}, 0) \mathrm{d} z+(1-\gamma)^{\delta} \int_{0}^{\infty} z^{\delta} f_{Z}(z ; \alpha, \tilde{\beta}, \tilde{\sigma}, 0) \mathrm{d} z \\
& =(1+\gamma)^{\delta} M_{s}\left(f_{Z}(-z ; \alpha, \tilde{\beta}, \tilde{\sigma}, 0)\right)+(1-\gamma)^{\delta} M_{s}\left(f_{Z}(z ; \alpha, \tilde{\beta}, \tilde{\sigma}, 0)\right),
\end{aligned}
$$

where $\delta=s-1$. The Mellin transform of H-function is well defined ( see, Mathai (2010)) by:

$$
\int_{0}^{\infty} x^{s-1} H_{p, q}^{m, n}\left[c z \mid \begin{array}{c}
\left(a_{p}, A_{p}\right) \\
\left(b_{q}, B_{q}\right)
\end{array}\right] d x=\Xi_{p, q}^{m, n}(s)(c z)^{-s}
$$

Thus, Inserting (3.5) and (3.6) in (3.13) we obtained

$$
M_{s}\left(f_{Z}(z ; \alpha, \tilde{\beta}, \tilde{\sigma}, 0)\right)=\frac{\tilde{\sigma}^{s-1} \Gamma(s) \Gamma\left(\frac{1-s}{\alpha}\right)}{\alpha \Gamma\left[\left(\frac{1}{2}+\tilde{\beta} \frac{k(\alpha)}{2 \alpha}\right)(1-s)\right] \Gamma\left[\frac{1}{2}-\tilde{\beta} \frac{k(\alpha)}{2 \alpha}+\left(\frac{1}{2}+\tilde{\beta} \frac{k(\alpha)}{2 \alpha}\right)(s)\right]},
$$

for $z \geq 0$, and

$$
M_{s}\left(f_{Z}(-z ; \alpha, \tilde{\beta}, \tilde{\sigma}, 0)\right)=\frac{\tilde{\sigma}^{s-1} \Gamma(s) \Gamma\left(\frac{1-s}{\alpha}\right)}{\alpha \Gamma\left[\left(\frac{1}{2}-\tilde{\beta} \frac{k(\alpha)}{2 \alpha}\right)(1-s)\right] \Gamma\left[\frac{1}{2}+\tilde{\beta} \frac{k(\alpha)}{2 \alpha}+\left(\frac{1}{2}-\tilde{\beta} \frac{k(\alpha)}{2 \alpha}\right)(s)\right]},
$$

for $z<0$. Substituting (3.14) and (3.15) in (3.12), we obtain (3.10). $\square$

Note that the expression in Corollary 3.1 of Diongue (2008) is a particular case of (3.10), he considered $\beta=0$.

In the following proposition, we obtain the $\delta$-moment for $Z \sim G E V(\xi, 1,0)$.

Proposition 2. If $Z \sim G E V(\xi, 1,0)$, then the delta moment $E(|Z|-\gamma Z)^{\delta}$ is given by:

$$
\frac{(1-\gamma)^{\delta}}{\xi^{\delta+1}} \sum_{k=0}^{\infty} \frac{(-1)^{k}}{k !} \frac{\Gamma(\delta+1) \Gamma\left(\frac{k+1}{\xi}-\delta\right)}{\Gamma\left(\frac{k+1}{\xi}+1\right)}+\frac{(1+\gamma)^{\delta}}{\xi^{\delta+1}} \sum_{k=0}^{\infty} \sum_{n=0}^{\infty} \frac{(-1)^{k}}{n ! k !} \frac{\Gamma\left(\frac{k+1}{\xi}+n+1\right)}{(\delta+n+1) \Gamma\left(\frac{k+1}{\xi}+1\right)}
$$

when $\xi>0$, and $\delta<\frac{1}{\xi}$ and by

$$
\frac{(1+\gamma)^{\delta}}{\xi^{\delta}} \sum_{k=0}^{\infty}(-1)^{k}\left(\begin{array}{l}
\delta \\
k
\end{array}\right)[\Gamma(1-\delta k)-\gamma(1-\xi n, 1)]+\frac{(1-\gamma)^{\delta}}{\xi^{\delta}} \sum_{k=0}^{\infty}(-1)^{k}\left(\begin{array}{l}
\delta \\
k
\end{array}\right) \gamma(\xi n-\xi \delta+1,1)
$$


when $\xi<0$ and $\delta>0$. The function $\gamma(\cdot, \cdot)$ is the incomplete Gamma and $\left(\begin{array}{l}\delta \\ k\end{array}\right)=\frac{\delta(\delta-1) \cdots(\delta-k+1)}{k !}$ is the binomial term.

Proof: When $\xi>0$, by (2.2),

$$
\begin{aligned}
E(|Z|-\gamma Z)^{\delta} & =(-1-\gamma)^{\delta} \int_{-1 / \xi}^{0} z^{\delta}(1+\xi z)^{-1-1 / \xi} \exp \left(-(1+\xi z)^{-1 / \xi}\right) \mathrm{d} z \\
& +(1-\gamma)^{\delta} \int_{0}^{\infty} z^{\delta}(1+\xi z)^{-1-1 / \xi} \exp \left(-(1+\xi z)^{-1 / \xi}\right) \mathrm{d} z
\end{aligned}
$$

In (3.18), inserting the representation $\exp \left(-(1+\xi z)^{-1 / \xi}\right)=\sum_{k=0}^{\infty} \frac{(-1)^{k}(1+\xi z)^{-k / \xi}}{k !}$ in the last integrals and changing the integration order,

$$
\begin{aligned}
E(|Z|-\gamma Z)^{\delta} & =(-1-\gamma)^{\delta} \sum_{k=0}^{\infty} \frac{(-1)^{k}}{k !}\left(\int_{-1 / \xi}^{0} z^{\delta}(1+\xi z)^{-\frac{k+1}{\xi}+1} d z\right) \\
& +(1-\gamma)^{\delta} \sum_{k=0}^{\infty} \frac{(-1)^{k}}{k !}\left(\int_{0}^{\infty} z^{\delta}(1+\xi z)^{-\frac{k+1}{\xi}+1} d z\right) .
\end{aligned}
$$

Now, substituting $\xi z$ for $u$ in (3.19),

$$
\begin{aligned}
E(|Z|-\gamma Z)^{\delta} & =\frac{1}{\xi}\left[\frac{1}{\xi}(-1-\gamma)\right]^{\delta} \sum_{k=0}^{\infty} \frac{(-1)^{k}}{k !}\left(\int_{-1}^{0} u^{\delta}(1+u)^{-\frac{k+1}{\xi}+1} d u\right) \\
& +\frac{1}{\xi}\left[\frac{1}{\xi}(-1-\gamma)\right]^{\delta} \sum_{k=0}^{\infty} \frac{(-1)^{k}}{k !}\left(\int_{0}^{\infty} u^{\delta}(1+u)^{-\frac{k+1}{\xi}+1} d u\right)
\end{aligned}
$$

To calculate the first integral of (3.20) we use the representation $(1+z)^{-b}=\sum_{n=0}^{\infty} \frac{\Gamma(b+n)}{\Gamma(b)} \frac{(-z)^{n}}{n !}$ valid for $|z|<1$ and $b \in \mathbb{R}^{+}$. However, to calculate the second integral we use the fact that $(1+u)^{-\frac{k+1}{\xi}+1}=$ $\frac{1}{\Gamma\left(\frac{k+1}{\xi}+1\right)} H_{1,1}^{1,1}\left[c z \mid \begin{array}{c}\left(1,-\frac{k+1}{\xi}\right) \\ (1,0)\end{array}\right]$ and then apply (3.13).

When $\xi<0$,

$$
\begin{aligned}
E(|Z|-\gamma Z)^{\delta} & =(1+\gamma)^{\delta} \int_{-\infty}^{0} z^{\delta}(1+\xi z)^{-1-1 / \xi} \exp \left(-(1+\xi z)^{-1 / \xi}\right) \mathrm{d} z \\
& +(1-\gamma)^{\delta} \int_{0}^{-1 / \xi} z^{\delta}(1+\xi z)^{-1-1 / \xi} \exp \left(-(1+\xi z)^{-1 / \xi}\right) \mathrm{d} z
\end{aligned}
$$

In (3.21), substituting $\left(-(1+\xi z)^{-1 / \xi}\right)$ for $u$, we obtained

$$
\begin{aligned}
E(|Z|-\gamma Z)^{\delta} & =\frac{(1+\gamma)^{\delta} 1^{\delta}}{\xi^{\delta}} \int_{1}^{\infty}\left(1-u^{-\xi}\right)^{\delta} \exp (-u) \mathrm{d} u \\
& +\frac{(1-\gamma)^{\delta} 1^{\delta}}{\xi^{\delta}} \int_{0}^{1}\left(u^{-\xi}-1\right)^{\delta} \exp (-u) \mathrm{d} u
\end{aligned}
$$

To evaluate the integrals in (3.22) we insert the binomial series $\sum_{n=0}^{\infty}\left(\begin{array}{c}\delta \\ n\end{array}\right)(-1)^{n} u^{-\xi n}=\left(1-u^{-\xi}\right)^{\delta}$ and $\sum_{n=0}^{\infty}\left(\begin{array}{c}\delta \\ n\end{array}\right)(-1)^{n}\left(u^{-\xi}\right)^{\delta-n}=\left(u^{-\xi}-1\right)^{\delta}$, then

$$
\begin{aligned}
E(|Z|-\gamma Z)^{\delta} & =\frac{(1+\gamma)^{\delta} 1^{\delta}}{\xi^{\delta}} \sum_{n=0}^{\infty}\left(\begin{array}{c}
\delta \\
n
\end{array}\right)(-1)^{n}\left(\int_{1}^{\infty} u^{-\xi n} \exp (-u) \mathrm{d} u\right) \\
& +\frac{(1-\gamma)^{\delta} 1^{\delta}}{\xi^{\delta}} \sum_{n=0}^{\infty}\left(\begin{array}{c}
\delta \\
n
\end{array}\right)(-1)^{n}\left(\int_{0}^{1} u^{-\xi \delta+\xi n} \exp (-u) \mathrm{d} u\right) .
\end{aligned}
$$

As the last integrals are incomplete gamma functions , the result (3.17) is obtained from (3.23). 
4. Application. In this section we used the GEVStableGarch routine to estimate ARMA-GARCH and ARMA-APARCH models to three dataset contains the returns from January 3, 2000 to January 3, 2015.

To use the GEV conditional distribution, we considered the monthly maximum returns of each data set.

TABLE 4.1

ARMA-GARCH and ARMA-APARCH with GEV conditional distribution.

\begin{tabular}{cccccc}
\hline Serie & Modelo & -LLH & AIC & AICc & BIC \\
\hline \multirow{2}{*}{ Microsoft } & ARMA(2,2)-GARCH(1,2) & -6826.20 & -13632.34 & -13632.25 & -13573.96 \\
& ARMA(2,2)-APARCH(1,2) & -7002.14 & -13982.28 & -13982.18 & -13918.06 \\
\multirow{2}{*}{ Google } & ARMA(1,2)-GARCH(1,1) & -5378.98 & -10741.97 & -10741.9 & -10696.52 \\
& ARMA(1,0)-APARCH(1,2) & -5458.77 & -10899.55 & -10899.47 & -10848.43 \\
\multirow{2}{*}{ S\&P500 } & ARMA(1,1)-GARCH(2,1) & -5862.58 & -11711.16 & -11711.07 & -11675.39 \\
& ARMA(1,1)-APARCH(2,2) & -5960.33 & -11898.65 & -11898.43 & -11842.44
\end{tabular}

Although ARMA-APARCH model has more parameters, according to the AIC and AICc criterions this model allowed us to better fit the data.

5. Conclusions. Explicit expressions of $\lambda_{i}$, where $Z_{t}$ has stable and GEV distributions, are obtained. They allow the accuracy of the estimation algorithms in the GEVStableGarch package.

6. Acknowledgements. We gratefully thank Dr. José Curto and Dr. John Frain for providing some of the data sets used in this work. The authors also wish to thank J. P. Nolan who kindly gave his Rstable package for computing stable densities in this work. The stable package is available at urlhttp://RobustAnalysis.com. S.R.C. Lopes research was partially supported by CNPq-Brazil and by INCT em Matemática.

References

[1] Bollerslev,T. and Ghysels, E. Autoregressive Conditional Heteroscedasticity, Journal of Business and Economic Statistics, 14 (1986), pp 139-151.

[2] Bougerol, P. and Picard, P. Stationarity of GARCH Processes and of some Non-Negative Time Series, Journal of Econometrics, 52 (1992), pp 115-127.

[3] Brockwell, P.J. Linear Prediction of ARMA Processes with Infinite Variance, Stochastic Processes and their Applications, 19 (1985), pp 281-296.

[4] Brockwell, P.J. Davis, R.A. Time Series: Theory and methods, Springer (1991).

[5] Brummelhuis, R. and Kaufmann, R. Time Scaling For GARCH(1,1) and AR(1)-GARCH(1,1) Processes, Journal of Risk, 2007, www.risk.net/journal-of-risk/technical-paper/2161017/time-scaling-value-risk-garch-ar-garch-processes.

[6] Curto, J.D. and Tavares, A.N. and Tavares, G.N. Modelling Heavy Tails and Asymmetry Using ARCH-type Models with Stable Paretian Distributions, Vol 51 (2008), Springer.

[7] Ding, Z. and Granger, C.W.J. and Engle, R.F. A Long Memory Property of Stock Market Returns and a New Model , Journal of Empirical Finance, 1 (1993), pp 83-106.

[8] Diongue, A.K. An investigation of Stable-Paretian Asymmetric Power GARCH Model, Journal des Sciences , 8 (2008), pp $15-26$.

[9] Engle, R.F. Autoregressive Conditional Heteroscedasticity with Estimates of the Variance of United Kingdom Inflation, Econometrica 50 (1982), pp 987-1007.

[10] Frain, J.C. Studies on the Application of the Alpha-stable Distribution in Economics , Phd Thesis-Trinity College, www.tcd.ie/Economics/staff/frainj/Stable-Distribution/thesis-main-5.pdf, (2009).

[11] Jenkinson, A.F. The frequency distribution of the annual maximum (or minimum) values of meteorological elements, Quarterly Journal of the Royal Meteorological Society, 81 (1955), pp 158-171.

[12] Jondeau, E.; Poon, S.H. and Rockinger, M. Financial Modeling under non-Gaussian Distributions, Springer Science \& Business Media , 2007.

[13] Ling, S. and McAleer, M. Necessary and Sufficient Moment Conditions for the GARCH(r,s) and Asymmetric Power GARCH( $r$,s) Models, Econometric Theory, 18(2002), pp 722-729.

[14] Mathai, A.M.; Saxena, R.K.; Haubold, H.J. The H-function: Theory and Applications, Springer-Verlag New York, (2010).

[15] Mittnik, S. and Panosrka, A.K. and Rachev, S. T. Stable GARCH Models for Financial Time Series, Applied Mathematical Letters, 8 (1995), pp 33-37.

[16] Mittnik, S. amd Paolella, M.C. Conditional Density and Value-At-Risk Prediction of Asian Currency Exchange Rates , ournal of Forecasting, 19 (2000), pp 313-333.

[17] Mittnik, S. and Paolella, M.S. and Rachev, S.T. Stationarity of Stable Power-GARCH Processes, Journal of Econometrics , 106 (2002), pp 97-107.

[18] Nakatsuma, T. Bayesian Analysis of ARMA-GARCH Models: A Markov Chain Sampling Approach, Journal of Econometrics, 95 (2000), pp 57-69.

[19] Nelson, D. Stationarity and Persistence in the GARCH(1,1) Model, Econometric Theory, 6 (1990), pp 318-344. 
[20] Nolan, J.P. Numerical Calculations of Stable Densities and Distribution Functions, Communication Statistics. Stochastic Models, 13 (1997), pp 759-774.

[21] Nolan, J.P. Stable Distributions Models for Heavy Tailed Data, Copyright, http:/ / www.math.ucla.edu/ biskup/275b.1.13w/ PDFs/Nolan.pdf (2009).

[22] Schneider, W.R. Stable distributions: Fox function representation and generalization, Lecture Notes in Physics, 262 (1986), pp 497-511.

[23] Sousa, T.R.; Otiniano, C.E.G. and Lopes, S.R.C. GEVStableGarch: An R Package for ARMA-GARCH or ARMA-APARCH Estimation with Conditional Stable and GEV Distributions, http://CRAN.R-project.org/package=GEVStableGarch, 2014.

[24] Wuertz,D.; Chalabi,Y. and Luksan, L. , Estimation of ARMA Models with GARCH/APARCH Errors: An R and SPlus Software Implementation, (2009),Journal of Sta- tistical Software, forthcoming. URL http://www-stat.wharton.upenn.edu/ steele/ Courses/956/RResources/GarchAndR/WurtzEtAlGarch.pdf.

[25] Zhao, X.; Scarrott, C.; Oxley, L.; Reale, M., Dependence in Extreme Value Models with Bayesian Inference, Mathematics and Computers in Simulation, 81 (2011), pp 1430-1440. 